\title{
Antibiotic Bone Cement Combined With Other Comprehensive Interventions in the Treatment of Diabetic Foot Osteomyelitis
}

\section{Fanyu Bu}

Wuxi Ninth People's Hospital: Wuxi Hand Surgery Hospital

\section{Xiaofeng Guo}

Wuxi Ninth People's Hospital: Wuxi Hand Surgery Hospital

\section{Peng Xu}

Wuxi Ninth People's Hospital: Wuxi Hand Surgery Hospital

Jin Wang

Wuxi Ninth People's Hospital: Wuxi Hand Surgery Hospital

Mingyu Xue

Wuxi Ninth People's Hospital: Wuxi Hand Surgery Hospital

XiaoYun Pan ( $\sim$ panxiaoyun89@suda.edu.cn )

Wuxi Ninth People's Hospital: Wuxi Hand Surgery Hospital

\section{Research article}

Keywords: Antibiotic bone cement, Diabetic foot, Osteomyelitis, Vancomycin, VSD

Posted Date: April 27th, 2021

DOI: https://doi.org/10.21203/rs.3.rs-431432/v1

License: () (1) This work is licensed under a Creative Commons Attribution 4.0 International License. Read Full License 


\section{Abstract}

\section{Background}

Diabetic foot osteomyelitis (DFO) is serious chronic complication that causes disability or death in diabetic patients. Antibiotic-loaded bone cement is an effective sustained-release system for the treatment of chronic osteomyelitis and induces biofilm formation. This study aimed to valuate the outcomes and summarize the experiences of bone cement loaded with vancomycin combined with other comprehensive interventions in the treatment of DFO.

\section{Methods}

One hundred and twelve involved feet in 93 patients (43-92 years old) with DFO treated with antibioticloaded bone cement combined with other comprehensive interventions were retrospectively analyzed. The durations of oral and intravenous antibiotics and hospitalization, ulcer healing times, recurrence and rehospitalization rates, and the rates of amputation above the ankle were evaluated at the last follow-up. One hundred and forty four pathogenic bacteria were co-cultured from the secretions of deep wounds from foot ulcers. The Maryland criteria were used to evaluate the recoveries of foot functions.

\section{Results}

Eighty seven patients with 105 involved feet were followed up successfully over an average period of 14 months. All wounds exhibited good union on follow up, and DFO was cured. The average durations of oral and intravenous antibiotic administrations were $12.2 \pm 1.5$ and $10.8 \pm 2.5$ days, respectively. The average duration of hospitalization was $14.0 \pm 2.7$ days and the healing time for the ulcers was $37.8 \pm$ 6.3 days. Rehospitalization presented in 21 (18.8区) foot ulcers among those with ulcer recurrence. No patients required amputation above the ankle. According to the Maryland criteria, 31, 45, 26, and three feet were rated as excellent, good, fair, and failures, respectively. Overall, $72 \%$ were rated as excellentgood.

\section{Conclusions}

The rate of amputation above the ankle was significantly reduced with the use of comprehensive interventions to retain foot function and improve quality of life. This management strategy in the treatment of DFO is effective and comprehensive comprehensive; therefore, it should be more frequently used in clinical settings.

\section{Introduction}

In the recent years, the prevalence of diabetes and thus the direct health expenditures resulting from diabetic have continued to rise globally $(1,2)$. There is a high incidence of diabetic foot, serious and hard to treat chronic complication leading to disability and even death among diabetic patients (3). Once diabetic foot osteomyelitis (DFO) occurs, treatment becomes even more challenging as the 
managements involves dealing with chronic ulcers, necrotic soft tissues and infected bones (4). Despite the wide variety of therapeutic strategies available for DFO, the rates of amputation and mortality remain high. DFO is challenging to diagnose early, leading to obvious bone destruction in clinical diagnosis. Additionally, there is still controversy regarding the best treatment, and whether antibiotics or surgery should be used (5).

In previous studies, antibiotics have been the primary method used for the conservative treatment of DFO, especially in cases with mild infection (6-8). The main advantage is that it does not change the structure of the foot, thus reducing the recurrence of ulcers caused by biomechanical changes. Antibiotics are also more economical and avoid the potential complications associated with surgical procedures (9). However, systematic antibiotics can hardly permeate the infective positions due to the biological membrane barriers produced by bacteria which make osteomyelitis uncurable. Moreover, most diabetic foot patients have vascular disease, resulting in blood drug concentrations at the infective lesion, thus inhibiting the ability to achieve anti-bacterial infection. Additionally, the rate of adverse drug reactions increases as the tolerance of bacteria increases when the durations of systematic antibiotics are exceeded (10).

Some experts believe that once osteomyelitis of diabetic foot has been diagnosed, it is predominantly a surgical disease (11). Thus, surgical operations, including debridement and varying degrees of amputation, are important methods of treating DFO. The operations can remove a large amount of planktonic bacteria by thoroughly cleaning the infected and necrotic tissues, and by promotion of rapid wound healing (12). However, this demands that potentially involved foot bone, skin, and soft tissue are widely excised to achieve a thorough debridement. This can sequentially damage the bone structure of the foot, causing changes in the biomechanical functionality of the lower limbs. Thereafter, it is possible that DFO and ulcers will recur due to the increased local stress on the sole of the foot, which thus drives the decision to perform an amputation. Existing studies have reported that 5-year mortality after limb amputation is approximately $47 \%$ and severe changes occur in approximately $50 \%$ of opposite limbs two years after a limb has been amputated (13). The prognosis for diabetic foot amputees is even worse than that for individuals with many malignancies (14). Therefore, systemic antibiotics alone or traditional surgery are not ideal treatments for DFO.

Since the 1970s, antibiotic-loaded bone cement has been widely used in the treatment of chronic traumatic osteomyelitis and bone nonunion and defects (15). This procedure releases high concentrations of antibiotics locally and enables bioactive membranes to promote wound healing. Despite several studies having reported promising outcomes for the use of this technique in the treatment of DFO, it remains challenging. Herein, we describe the use of a combination of antibiotic bone cement and other interventions for the treatment of DFO. Additionally, we evaluated the outcomes and summarized our experiences using this treatment for DFO.

\section{Methods}




\section{Patients}

One hundred and twelve involved feet in 93 patients with DFO treated with antibiotic-loaded bone cement from March 2016 to December 2018 were retrospectively analyzed. All patients had a strong desire for limb salvage. The clinical data were recorded, including general clinical and demographic information, Wagner classification, durations of oral and intravenous antibiotics, duration of hospitalization, and time until the ulcer healed. The diagnosis of DFO was confirmed using presurgical X-rays and computed tomographs (CTs) of the involved foot. Narrowness and occlusions in the chief arteries were affirmed in the involved limbs using ultrasonography and CT of the arteries in both lower extremities.

\section{Basic treatment}

General condition and nutritional status of the patients were adjusted to meet the requirements of the operation after hospitalization. Fasting plasma glucose and 2h-PG were controlled below 8.0-8.5 $\mathrm{mmol} / \mathrm{L}$ and $12 \mathrm{mmol} / \mathrm{L}$, respectively. Blood pressure was maintained below 140/80 $\mathrm{mmHg}$. LDL-C was controlled below $1.8 \mathrm{mmol} / \mathrm{L}$. Operations were planned after consultations with the concerned departments. Secretions from beside the wound edges were acquired for bacterial culturing to provide a reference for selecting the best anti-infection treatment.

\section{Surgical procedures}

The patients adopted the supine position and the area above the knee was disinfected after spinal anesthesia or general tracheal intubation. It is important to note that the use of a tourniquet was not advised. The wound was cleaned with large quantities of iodophor, mixed with hydrogen peroxide solution and physiological saline. Proliferative inflammatory granulation was cleaned, as well as the necrotic skin, foot bone, articular capsule with the ligament, the tendon, and the fascia. Skin and muscle with possible vitality were retained. Secretions at the boundary of the active and necrotic tissues and bone tissues were acquired during the operation for bacterial culturing. Based on the findings from the cultured samples, the antibiotic treatment was adjusted accordingly. Debridement was conducted until the wound surface was fresh with a small flow of blood. The wound was washed with large quantities of iodophor mixed with hydrogen peroxide solution and thereafter physiological saline again. Bone cement was mixed with vancomycin in a ratio of $2-3 \mathrm{~g}$ to $40 \mathrm{~g}$ and was then cooled down with physiological saline. Antibiotic-loaded bone cement was remodeled to fill into the wound lacuna. Skin and soft tissue around the wound were tightened and sutured up. Importantly, there was no need to wrap the bone cement wholly but it was important to ensure that no dead space remained. Silver ions dressing or vacuum sealing drainage (VSD) was pasted to the wound for protection and then the involved leg was supported with a plaster.

\section{Postoperative management}

Wound dressing was performed every other day (VSD would be removed one week later) post operation. Intravenous antibiotics were replaced by oral antibiotics when the wounds exhibited initial healing with no effusion 7-10 days post operation. The patients were then discharged from the hospital with 
education provided by doctors on key requirements for wound nursing and sterile maintenance. The patients were then instructed to change the dressings by themselves and continue controlling their blood sugar, engage in appropriate functional training, and avoid bearing weight on the recovering legs. A second operation to remove the bone cement and close the wound was conducted 3-5 weeks after the first operation. The exposed bone was then covered with the subcutaneous fascia tissue. Interrupted sutures were placed on the skin of the wound margin if no tension was observed. Skin grafting from the groin was used if the tensile force was too high to suture. As the wound healed and/or the grafted skin survived the patient was allowed to leave the hospital and oral antibiotics were stopped. Patients were instructed to wear customized, fitted orthopedic shoes with soft soles to disperse the pressure under their foot and they were instructed to regulate their blood sugar reasonably. The recovery of the foot wound was examined are every follow-up.

\section{Follow-up and evaluation}

Followed-up was conducted regularly after surgery. DFO was considered to be cured if the signs of infection in the involved bones had subsided, as affirmed from wound stabilization or improvement observed radiographically, and the C-reactive protein, erythrocyte sedimentation rate, and procalcitonin levels were all within the normal range, and that the wound had healed well with no ulcers and symptoms of swelling or pain, indicating that all local and systematic signs of infection had disappeared. The durations of oral and intravenous antibiotics and hospitalization, time required for the ulcer to heal (from the first operation to the time of ulcer healing after the second operation), the ulcer recurrence and rehospitalization rates, and the rate of amputations above the ankle were evaluated at the last follow-up. The Maryland criteria ${ }^{12}$ were used to evaluate the recovery of foot functions.

\section{Statistical analysis}

All analyses were conducted using SPSS 24.0.

\section{Results}

Overall, there were 62 involved feet in 57 men and 50 in 36 women; the average age at operation was 67 years. On average, the included patients had 17 year (3-35) and 15 month (2-25) histories of diabetes and foot ulcers. Seventy seven, 65 , and 61 patients had heart diseases, retinopathy, and peripheral vascular disease in their lower extremities, respectively. Based on the Wagner classification, 67, 41, and four feet were type 3,4 , and 5 , respectively (Table 1 ). 
Table 1

Patients

\begin{tabular}{|ll|}
\hline Characteristic & Value(\%) \\
\hline Sex & \\
\hline Male & $57(61.29 \%)$ \\
Female & $36(38.71 \%)$ \\
\hline Mean age (y) & 67 \\
\hline Wagner classification & $67(59.82 \%)$ \\
Type III & $41(36.61 \%)$ \\
Type IV & $4(3.57 \%)$ \\
Type V & \\
\hline Heart diseases & $77(82.80 \%)$ \\
\hline Retinopathy & $65(69.89 \%)$ \\
\hline Peripheral vascular disease & $61(65.59 \%)$ \\
\hline
\end{tabular}

In the evaluation of 112 diabetic feet, 144 pathogenic bacteria were co-cultured from the secretions of deep wounds of foot ulcers. There were 56 cases of Gram-positive (G+) bacterial infections, including eight cases of Methicillin-resistant Staphylococcus aureus (MRSA), 79 cases of Gram-negative (G-) bacterial infections, and nine cases of fungal infection, among which 23 cases were mixed infection. There were 89 cases of single bacterial infection, 24 cases of two bacterial infections, and nine cases of three bacterial infections. Staphylococcus aureus was the most common type of infection, followed by Pseudomonas aeruginosa and Coagulase negative Staphylococcus aureus (Table 2). 
Table 2

Pathogenic bacteria types, strains and constituent ratios of secretion specimens

\begin{tabular}{|lll|}
\hline Bacteria & Strains & Constituent ratio(\%) \\
\hline G+ & & \\
\hline Staphylococcus aureus & 24 & 16.67 \\
\hline Coagulase negative staphylococcus & 16 & 11.11 \\
\hline Streptococci & 5 & 3.47 \\
\hline Enterococci & 11 & 7.64 \\
\hline G- & & \\
\hline Pseudomonas aeruginosa & 18 & 12.50 \\
\hline Enterobacter cloacae & 11 & 7.64 \\
\hline Escherichia coli & 9 & 6.25 \\
\hline Other Bacillus & 31 & 21.53 \\
\hline Monas & 10 & 6.94 \\
\hline Fungus & 9 & 6.25 \\
\hline Total & 144 & 100 \\
\hline
\end{tabular}

Overall, two patients died of cardio-cerebrovascular disease post-discharge and four patients were lost to follow-up. Eighty seven patients with 105 involved feet were followed up successfully over an average period of 14 months (6-25). All wounds exhibited good union on follow-up, and DFO was cured. The average durations of oral and intravenous antibiotic administration were $12.2 \pm 1.5$ days (10-14) and $10.8 \pm 2.5$ days (7-14), respectively. The average duration of hospitalization was $14.0 \pm 2.7$ days (1019). The average time required for the healing of the ulcer was $37.8 \pm 6.3$ days (27-49). Rehospitalization was required for 21 (18.8\%) foot ulcers as a result of ulcer recurrence. No patient required amputation above the ankle. Based on the Maryland criteria, 31, 45, 26, and three feet were classified as excellent, good, fair, and failures, respectively. Overall, $72 \%$ were classified as excellent-good.

\section{Typical case}

A 60-year-old man with a 16-year history of diabetes usually self-injected insulin to control his blood sugar, but it was still unstable. One month before the operation, the patient's heel was accidentally abraded by wearing leather shoes. At the time, only disinfection was performed. However, the wound showed no sign of recovery and gradually worsened. Upon presentation at the Department of Endocrinology in our hospital for anti-infection and blood glucose control treatment, the wound was filled with pus and had a peculiar smell. There were two ulcer wounds on the lateral heel of the right foot that 
were as deep as the bone $\left(1 \times 1 \mathrm{~cm}^{2}\right.$ and $4 \times 6 \mathrm{~cm}^{2}$, respectively). Additionally, the wound periphery was red and swollen (Figs. 1A, B). Physical examination revealed obvious dorsal foot pulse and numbness at the toe end. After the blood glucose and pressure were stabilized, debridement was performed. An incision was made along the lateral sinus of the foot through the plantar to the inside of the foot. Inflammatory tissue was observed to spread along the plantar aponeurosis to the distal plantar and inside the foot, and part of the calcaneal bone was corroded. The necrotic tissue was thoroughly removed by debridement along the wound margin, and incisional lavage and drainage for osteomyelitis and the abscess were performed (Fig. 1C). The wound was sutured after filling the medullary cavity with vancomycin bone cement (Figs. 1D, E). Bone cement removal surgery was performed after three weeks. The skin on the sole was partially necrotic. Fortunately, the blood supply within the wound was abundant, with new granulation tissue and no infection (Figs. 1F, G). After removing the necrotic skin, skin of full-thickness on the anterolateral thigh of the same side was grafted to cover the wound (Figs. $1 \mathrm{H}, \mathrm{I})$. The skin graft completely survived one week after operation and the wound healed after three months (Figs. 1J-L).

\section{Discussion}

Different from hematogenous osteomyelitis, the occurrence of DFO is mainly caused by the continuous invasion of bacteria in the infected soft tissue into the bone through bone exposure or fistula formed by diabetic foot ulcers. It mainly involves the weight-bearing parts of the foot (16). The development of diabetic foot is the result of a combination of peripheral vascular disease, diabetic peripheral neuropathy (DPN), and infection. DPN plays a major role in the etiology of diabetic foot, affecting the function of motor, sensory, and autonomic nerves. This not only leads to the loss of pain, temperature, pressure, and other protective sensations, but also leads to foot trauma and subsequent infection due to the undue pressure exerted on the feet without the patient's knowledge. Additionally, peripheral vascular diseases lead to poor blood supply which results in local drug concentrations that are ineffective against invading bacteria at the local region of infection. Infection then occurs once the bacteria invade and colonize. Healing becomes difficult if the above mentioned conditions are met. For the management of DFO, antiinfection and stabilization of the foot are the most important factors. Studies have shown that the effects of systemic antibiotics and traditional surgery on DFO are very limited. Adjunctive treatment of such infections with the use of local antibiotic delivery systems has shown improved outcomes (17).

Antibiotic bone cement was first used by Buchholz in 1970 (15), and since then, it has been commonly used as an effective adjuvant therapy for chronic infection, traumatic osteomyelitis, and nonunion (15, 18). Compared to systemic administration, local antibiotic therapy can avoid systemic adverse reactions with a longer duration and more accurate positioning (4). Possible residual bacteria post debridement could be killed by high concentrations of antibiotics released locally, for which the effective concentrations might be 1000 times that of systematic antibiotics. However, the dose of local vancomycin that passes into circulation is far less than that from systematic administration resulting in a mild presentation of adverse reactions. Conversely, it is not affected by ischemia of the limb as it acts directly at the lesion site. Additionally, the residual dead space post debridement can be filled with bone 
cement shaped properly in order to decrease the recurrence of infection and provide some leeway space, in the case of contracture of the skin margin. Recently, Li et al. investigated the role of induced membrane (IM) using antibiotic bone cement; they found that IM assisted the wound healing by promoting angiogenesis (19).

Currently, local drug-loading sustained-release systems can be divided into biodegradable and nonbiodegradable systems. Although biodegradable sustained-release systems have been an attractive treatment option in recent years, most have been used in animal studies. Therefore, polymethylmethacrylate (PMMA), a representative of non-degradable materials, has remained the standard for sustained-release carriers in the clinic. In this study, PMMA was chosen as the carrier because PMMA pellets can provide immediate stabilization or assist in maintaining the stabilization of the bone tissue structure. Additionally, it is beneficial to maintain the length of the bone and soft tissue in the area with the bone defect after debridement of the chronic osteomyelitis infected lesions, and to provide a good bone graft space and bed for the bone graft reconstruction of stage II bone defects.

For the treatment of osteomyelitis infection, it is critical to choose effective antibiotics. Although the distribution of pathogenic bacteria in DFO wounds has changed over the years, Staphylococcus aureus remains the most common infecting organism (20), as observed in the current study. More remarkably, the occurrence of multidrug-resistant organism isolation increases annually. MRSA as the most important of these pathogens, has been associated with a significantly higher rate of treatment failure in patients with diabetic foot infections (21). Vancomycin is a glycopeptide antibiotic, which has good antimicrobial activity against $\mathrm{G}+$ bacteria, especially for the treatment of MRSA. Additionally, the release curve of this drug in bone cement is stable, proving its stability to heat and its water-solubility.

In this study, DFO was treated using comprehensive interventions, including VSD and flap transplantation, instead of just a single application of antibiotic-loaded bone cement. VSD is reportedly safer and more effective than traditional treatments for patients with DFO (22). Complete drainage can be achieved under negative pressure (23); bacteria, pus, and exculpated necrotic tissue in the drainage area can be periodically and thoroughly drained from the body, and in the process the wound is kept completely clean. Furthermore, VSD reduces the degree of inflammation and improves phantom limb edema by reducing vascular permeability through aspiration. Conversely, the application of VSD in the negative pressure state can significantly expand the blood vessel diameter, improve blood flow velocity and blood flow, slightly promote capillary regeneration, and improve the partial pressure of oxygen in the foot tissue. In our study, antibiotic bone cement combined with VSD was used after complete debridement, and skin grafting or flap transplantation was performed in the second stage after the wound surface was stabilized. The average duration of treatment with antibiotics was 23.0 days and the average time in which ulcers healed was 37.8 days; these findings were shorter than those reported in some studies (24). All the cases in our study were cured and no amputations above the ankle were required. Based on the Maryland criteria, the excellent-good rare was $72 \%$. 
It is essential that comprehensive treatments control basic diseases for a sustained duration. In our experience, only the basic conditions are well-regulated if the patients can tolerate surgery and if the surgical outcomes can be sustained for long periods. Wounds should not be covered with tensile force or slap to avoid the injuries associated with ischemia in microcirculation. Skin grafting is sometimes possible. The use of customized and fitted orthopedic shoes with soft soles could disperse the pressure under the foot and improve the weight-bearing abilities in the lower limbs after the wound has healed. Exercises focused on protected function could improve the quantity of life and reduce the risk of amputation. However, not all patients can persist wearing the orthopedic shoes. Regular follow-up and coordination among patients' family members might assist them in avoiding the condition.

\section{Conclusions}

The short-term effects of antibiotic bone cement combined with other comprehensive interventions for the treatment of DFO were significant. Complete debridement was performed in the first stage, and vancomycin bone cement was implanted into the medullary cavity, followed by adequate drainage with VSD dressings. The second stage entailed wound closure, combined with the application of systemic sensitive antibiotics, for patients with severe skin defects and bone exposure, this was combined with skin flap transplantation to close the wound. This surgical plan can significantly shorten the treatment period for patients. Additionally, patients were empowered to care for their own wounds, thus requiring shorter durations of hospitalization. Finally, the need for amputation was reduced significantly with the use of comprehensive interventions to retain the function of the foot and improve the quality of life. This is an ideal choice for the clinical treatment of DFO and should be considered in clinical practice more regularly moving forward.

\section{List Of Abbreviations}

DFO, diabetic foot osteomyelitis; CT, computed tomography; VSD, vacuum sealing drainage; MRSA, Methicillin-resistant Staphylococcus aureus; DPN, diabetic peripheral neuropathy; IM, induced membrane; PMMA, polymethylmethacrylate

\section{Declarations}

\section{Acknowledgments}

No

\section{Author contributions}

Mingyu Xue and Xiaoyun Pan designed the study, collected the clinical data, and completed the manuscript. Fanyu Bu performed the operations. Xiaofeng Guo, Peng Xu and Jin Wang participated in the operations. All authors have no conflicts of interest to disclose. All authors have read and approved the final submitted manuscript. 


\section{Funding}

This work was supported by Top medical expert team of "Taihu Talent Program" in Wuxi (2020)

\section{Availability of data and materials}

The datasets used and analyzed during the current study are available from the corresponding author on reasonable request.

\section{Ethics approval and consent to participate}

The study protocol was approved by the ethics committee of The Ninth People's Hospital of Wuxi affiliated to Soochow University.

\section{Consent for publication}

Not applicable.

\section{Competing interests}

All authors have no conflicts of interest to disclose. All authors have read and approved the final submitted manuscript.

\section{Contributor Information}

Fanyu Bu, E-mail: Bufanyu1020@163.com

Xiaofeng Guo, Email: 15052178511@126.com

Peng Xu, Email: 17501488002@163.com

Jin Wang, Email: wangjin0311@qq.com

Mingyu Xue, E-mail: xuemingyu1010@sina.com

Xiaoyun Pan, Email: panxiaoyun89@suda.edu.cn

\section{References}

1. Saeedi P, Petersohn I, Salpea P, Malanda B, Karuranga S, Unwin N, et al. Global and regional diabetes prevalence estimates for 2019 and projections for 2030 and 2045: Results from the International Diabetes Federation Diabetes Atlas, 9(th) edition. Diabetes Res Clin Pract. 2019;157:107843.

2. Williams R, Karuranga S, Malanda B, Saeedi P, Basit A, Besancon S, et al. Global and regional estimates and projections of diabetes-related health expenditure: Results from the International Diabetes Federation Diabetes Atlas, 9th edition. Diabetes Res Clin Pract. 2020;162:108072. 
3. Giurato L, Meloni M, Izzo V, Uccioli L. Osteomyelitis in diabetic foot: A comprehensive overview. World J Diabetes. 2017;8(4):135-42.

4. Qin $\mathrm{CH}$, Zhou $\mathrm{CH}$, Song $\mathrm{HJ}$, Cheng GY, Zhang HA, Fang J, et al. Infected bone resection plus adjuvant antibiotic-impregnated calcium sulfate versus infected bone resection alone in the treatment of diabetic forefoot osteomyelitis. BMC Musculoskelet Disord. 2019;20(1):246.

5. Lipsky BA. Treating diabetic foot osteomyelitis primarily with surgery or antibiotics: have we answered the question? Diabetes Care. 2014;37(3):593-5.

6. Senneville E, Robineau O. Treatment options for diabetic foot osteomyelitis. Expert Opin Pharmacother. 2017;18(8):759-65.

7. Mutluoglu M, Lipsky BA. Diabetic foot osteomyelitis. CMAJ. 2016;188(17-18):E535.

8. Acharya S, Soliman M, Egun A, Rajbhandari SM. Conservative management of diabetic foot osteomyelitis. Diabetes Res Clin Pract. 2013;101(3):e18-20.

9. Game FL, Jeffcoate WJ. Primarily non-surgical management of osteomyelitis of the foot in diabetes. Diabetologia. 2008;51(6):962-7.

10. Tone A, Nguyen S, Devemy F, Topolinski H, Valette M, Cazaubiel M, et al. Six-week versus twelve-week antibiotic therapy for nonsurgically treated diabetic foot osteomyelitis: a multicenter open-label controlled randomized study. Diabetes Care. 2015;38(2):302-7.

11. Aragon-Sanchez FJ, Cabrera-Galvan JJ, Quintana-Marrero Y, Hernandez-Herrero MJ, Lazaro-Martinez $\mathrm{JL}$, Garcia-Morales E, et al. Outcomes of surgical treatment of diabetic foot osteomyelitis: a series of 185 patients with histopathological confirmation of bone involvement. Diabetologia. 2008;51(11):1962-70.

12. Lazaro-Martinez JL, Aragon-Sanchez J, Garcia-Morales E. Antibiotics versus conservative surgery for treating diabetic foot osteomyelitis: a randomized comparative trial. Diabetes Care. 2014;37(3):78995.

13. Moulik PK, Mtonga R, Gill GV. Amputation and mortality in new-onset diabetic foot ulcers stratified by etiology. Diabetes Care. 2003;26(2):491-4.

14. Allemani C, Weir HK, Carreira H, Harewood R, Spika D, Wang XS, et al. Global surveillance of cancer survival 1995-2009: analysis of individual data for 25,676,887 patients from 279 population-based registries in 67 countries (CONCORD-2). Lancet. 2015;385(9972):977-1010.

15. Wentao Z, Lei G, Liu Y, Wang W, Song T, Fan J. Approach to osteomyelitis treatment with antibiotic loaded PMMA. Microb Pathog. 2017;102:42-4.

16. Malhotra R, Chan CS, Nather A. Osteomyelitis in the diabetic foot. Diabet Foot Ankle. 2014;5.

17. Ramanujam CL, Zgonis T. Antibiotic-loaded cement beads for Charcot ankle osteomyelitis. Foot Ankle Spec. 2010;3(5):274-7.

18. Hake ME, Young H, Hak DJ, Stahel PF, Hammerberg EM, Mauffrey C. Local antibiotic therapy strategies in orthopaedic trauma: Practical tips and tricks and review of the literature. Injury. 2015;46(8):1447-56. 
19. Kavanagh N, Ryan EJ, Widaa A, Sexton G, Fennell J, O'Rourke S, et al. Staphylococcal Osteomyelitis: Disease Progression, Treatment Challenges, and Future Directions. Clin Microbiol Rev. 2018;31(2).

20. Barwell ND, Devers MC, Kennon B, Hopkinson HE, McDougall C, Young MJ, et al. Diabetic foot infection: Antibiotic therapy and good practice recommendations. Int J Clin Pract. 2017;71(10).

21. Kosinski MA, Lipsky BA. Current medical management of diabetic foot infections. Expert Rev Anti Infect Ther. 2010;8(11):1293-305.

22. Huang Q, Wang JT, Gu HC, Cao G, Cao JC. Comparison of Vacuum Sealing Drainage and Traditional Therapy for Treatment of Diabetic Foot Ulcers: A Meta-Analysis. J Foot Ankle Surg. 2019;58(5):954-8.

23. Yang H, Liu L, Li G, Chen Y, Jiang D, Wang W, et al. Growth Promoting Effect of Vacuum Sealing Drainage in the Healing Processes of Diabetic Foot Ulcers. Ther Clin Risk Manag. 2021;17:65-71.

24. Ulcay A, Karakas A, Mutluoglu M, Uzun G, Turhan V, Ay H. Antibiotherapy with and without bone debridement in diabetic foot osteomyelitis: A retrospective cohort study. Pak J Med Sci. 2014;30(1):28-31.

\section{Figures}



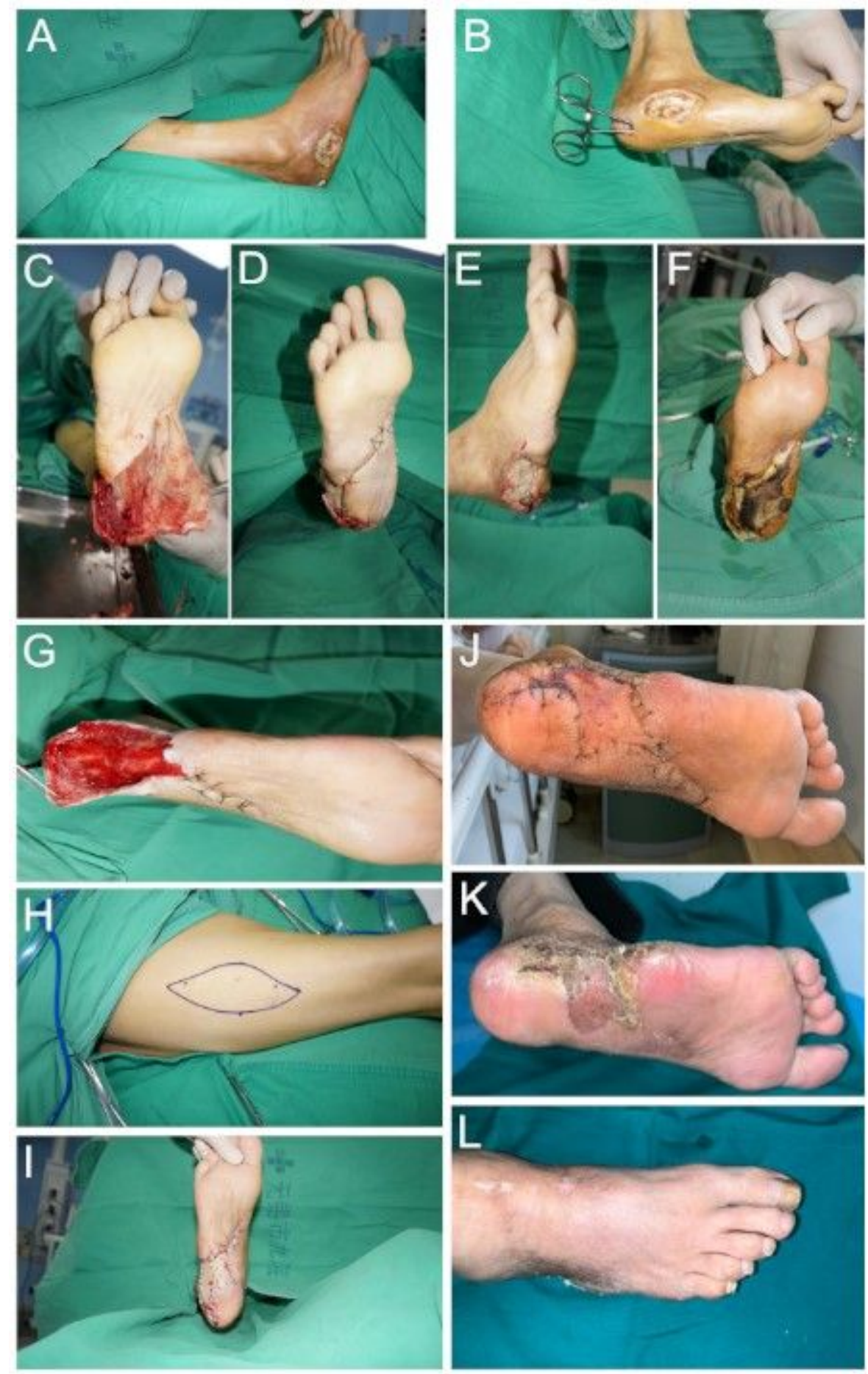

\section{Figure 1}

Typical case. A 60-year-old man with a 16-year history of diabetes. A,B: The size of the two ulcer wounds on the lateral heel of the right foot were $1 \times 1 \mathrm{~cm} 2$ and $4 \times 6 \mathrm{~cm} 2$, respectively. They were as deep as bone with discharging pus and fluid. C: Complete debridement. D, E: Filling the medullary cavity with vancomycin bone cement. F, G: Bone cement removal surgery was performed after three weeks. H, I: The wound was covered with a skin graft of full thickness. J: The skin graft completely survived one week after the operation. K, L: Appearance and healing three months after surgery. 